\title{
Treatment of Intracranial Meningioma in an Elderly Patient by Endovascular Embolization Without Subsequent Surgical Removal
}

\author{
Masanori SUZUKI ${ }^{1)}$ Shushi KOMINAMI ${ }^{1)}$ Shiro KOBAYASHI ${ }^{1)}$ Akira TERAMOTO $^{2)}$ \\ 1) Department of Neurosurgery, Nippon Medical School Chibahokusou Hospital \\ 2) Department of Neurosurgery, Nippon Medical School
}

\begin{abstract}
Objective: Intracranial meningioma surgery in the elderly sometimes causes neurological complications that significantly degrade quality of life. We report a case of intracranial meningioma that was effectively and safely treated by endovascular embolization using liquid materials without subsequent surgical removal. Case presentation: Our case of intracranial meningioma was in an 86-year-old woman treated by endovascular embolization using ethanol and n-butyl cyanoacrylate. Six days after embolization, preoperative neurological deficits were improved, no cognitive deterioration was detected, and tumor shrinkage was evident on brain magnetic resonance imaging. Tumor size remains unchanged two years after embolization.

Conclusion: Endovascular embolization using liquid materials may be safe for symptomatic meningioma in elderly patients to prevent various complications associated with surgical removal.
\end{abstract}

Key Words

ethanol, endovascular embolization, intracranial meningioma

<Correspondence Address : Masanori SUZUKI, 1715 Kamakari, Inzai City, Chiba, Japan E-mail: gorigori@r4.dion.ne.jp>

(Received March 9, 2012 : Accepted September 28, 2012)

\section{Introduction}

Meningioma is generally benign, and surgical removal is recognized as the best treatment option. However, elderly patients are more prone to disabling neurological deficits following surgery, including cognitive dysfunction. Surgical removal of meningioma may be safe for elderly patients with good general health and neurological status $^{8,10,18,22)}$. Several risk factors for postsurgical morbidity and mortality have been defined, including peritumoral edema, tumor location, tumor size, high preoperative American Society of Anesthesiology (ASA) physical status classification score, and low preoperative Karnofsky Performance Scale (KPS) index. Few studies of surgical meningioma treatment in the elderly have examined postoperative cognitive dysfunction and assessed the quality of life by the modified Rankin Scale (mRS). This report presents the case of an 86-year-old woman, with radiographically suspected meningioma, treated solely by endovascular embolization using ethanol and n-butyl-cyanoacrylate (NBCA).

\section{Case Presentation}

An 86-year-old woman was admitted to our department for progressive right hemiparesis including the face. Motor weakness gradually worsened during the first week after admission, and she developed right hemiplegia. Her general health condition was classified as ASA II and her neurological status was 50 on the KPS. She scored 27 (normal) on the mini-mental state examination (MMSE). Gadolinium-enhanced brain magnetic resonance imaging (MRI) revealed a clear mass with heterogeneous enhancement attached to the superior sagittal sinus and a dural tail sign in the falx cerebri (Fig. 1). Cerebral angiography indicated dural blood supply from the right middle meningeal artery, and pial blood 

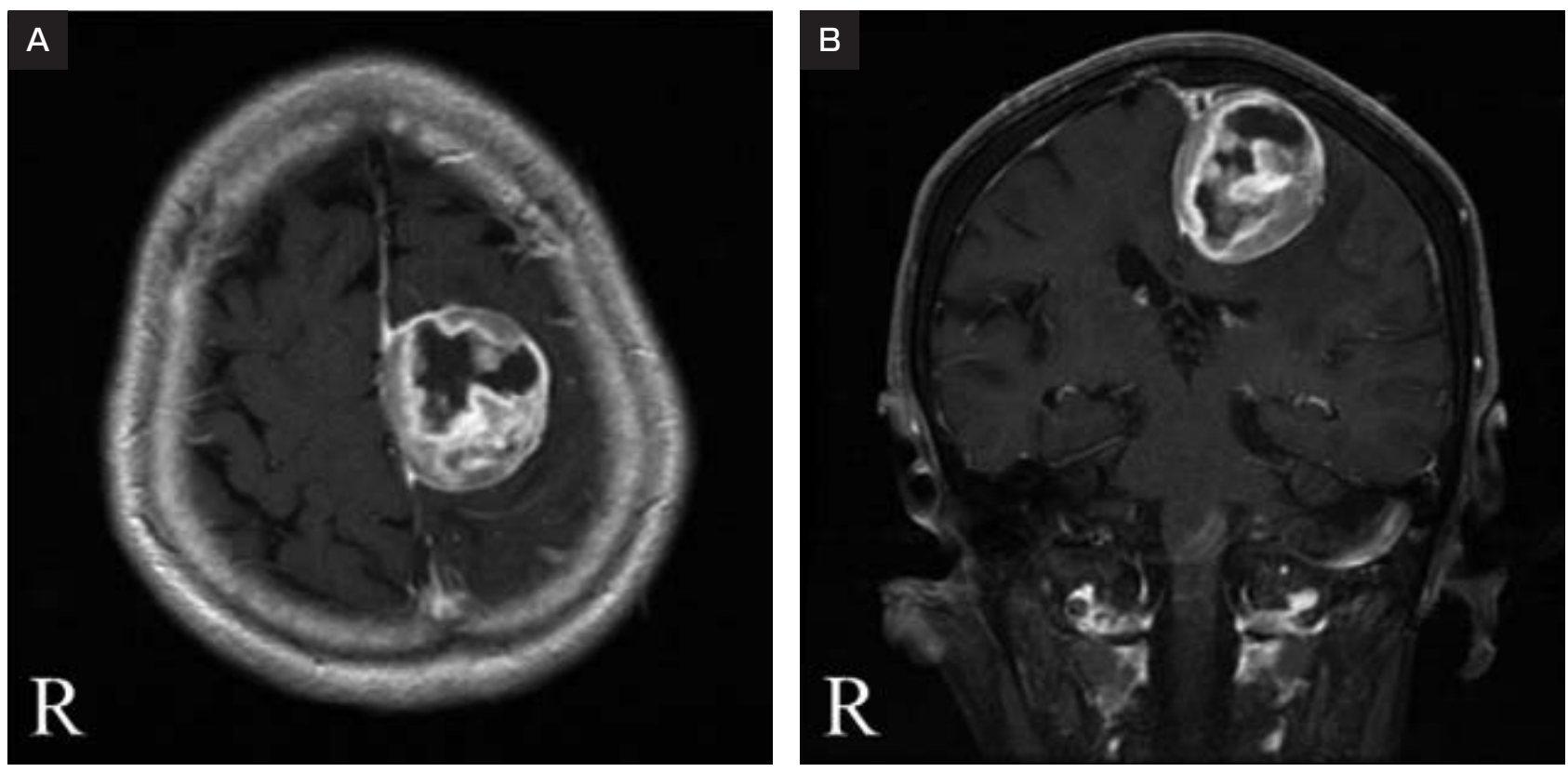

Fig. 1

Preoperative axial (A) and coronal (B) T1-weighted magnetic resonance images with contrast medium show a tumor with dural tail sign and peritumoral edema. Tumor contrast enhancement is heterogeneous because of intratumoral necrosis.
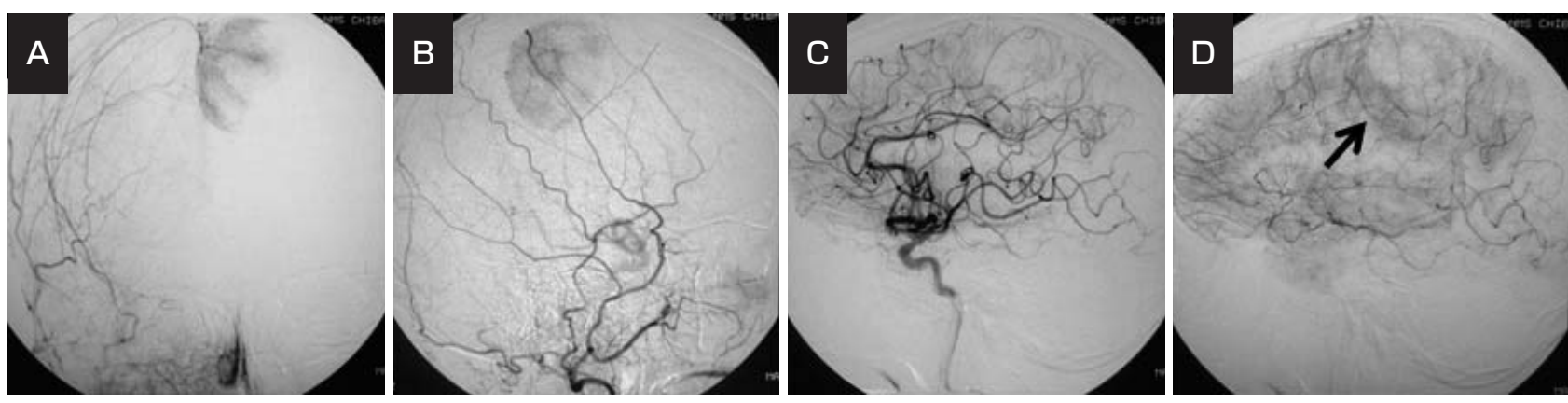

Fig. 2

Right external carotid angiograms. (A, B) (A) Anteroposterior view and (B) Lateral view show tumor stain entering from the middle meningeal artery.

Left internal carotid angiograms. (C, D) Lateral views of the early arterial phase (B) and late arterial phase (C) show pial blood supply (arrow).

supply from the left anterior cerebral artery (Fig. 2). We strongly suspected intracranial parasagittal meningioma based on radiological findings and performed endovascular embolization to induce tumor shrinkage rather than excision because open surgery could have caused postoperative complications, including motor weakness and memory disturbances. As embolic agents, ethanol was used to facilitate the penetration of the capillaries and NBCA was used to prevent recanalization and revascularization from the proximal artery. Under local anesthesia and sedation induced by $15 \mathrm{mg}$ of pentazocine and $25 \mathrm{mg}$ of hydroxyzine, a $5 \mathrm{Fr}$ introducer sheath was placed in the right femoral artery, a $5 \mathrm{Fr}$ Guider TM guiding catheter (Boston Scientific, Fremont, California, USA) was inserted in the right external carotid artery, and a Magic TM catheter (BALT, Montmorency, France) was positioned at the distal portion of the anterior branch of the right middle meningeal artery, at a site proximal to the falx cerebri. Lidocaine $4 \mathrm{mg} / \mathrm{ml}(0.4 \%)$ was injected into the feeding 

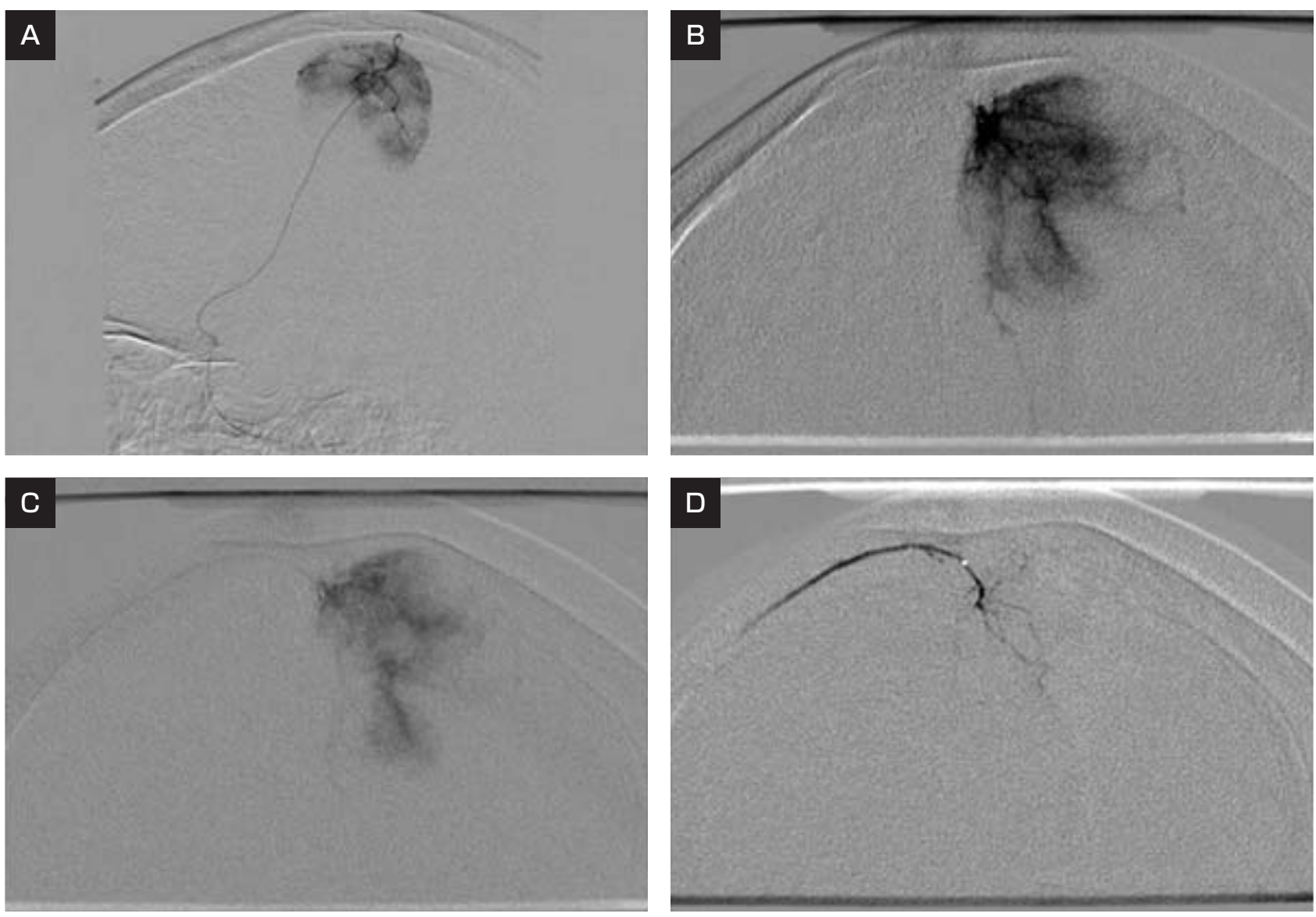

Fig. 3

Superselective angiograms from a microcatheter in the right middle meningeal artery at a site proximal to the falx cerebri.

A : Lateral view.

B : Anteroposterior view.

C : The tumor exhibits gradually reduced enhancement during ethanol and contrast medium injection.

$\mathrm{D}$ : Injection of n-butyl cyanoacrylate at the distal part of the middle meningeal artery.

artery to prevent severe local pain due to ethanol injection. A mixture of ethanol and contrast medium (2:1) was slowly and repeatedly injected into the tumor. Tumor enhancement gradually decreased during ethanol injection, and injection was terminated when the enhancement had almost disappeared. The total volume of ethanol mixture injected was about $1 \mathrm{ml}$. Finally, a 20\% NBCA-lipiodol mixture was injected into the tumor and carefully injected into the distal part of middle meningeal artery to avoid embolic complications (Fig. 3). After ethanol injection, the patient complained of mild headache without nausea and vomiting, but symptoms improved by the next day. Six days after embolization, her hemiparesis had already begun to improve as evidenced by a manual muscle test (MMT) score of 3, and brain MRI revealed tumor shrinkage. She retained preoperative cognitive function (postoperative MMSE score of 26) and was transferred to another hospital for rehabilitation one week after embolization without complication. Her hemiparesis had nearly disappeared by 3 months after embolization, and she has been visiting our hospital as an outpatient for the past 2 years. Her current neurological status is 80 on the KPS and 2 on the mRS. As shown in Fig 4, peritumoral edema gradually abated and the tumor regressed by $75 \%$.

\section{Discussion}

Preoperative embolization of intracranial meningiomas is 

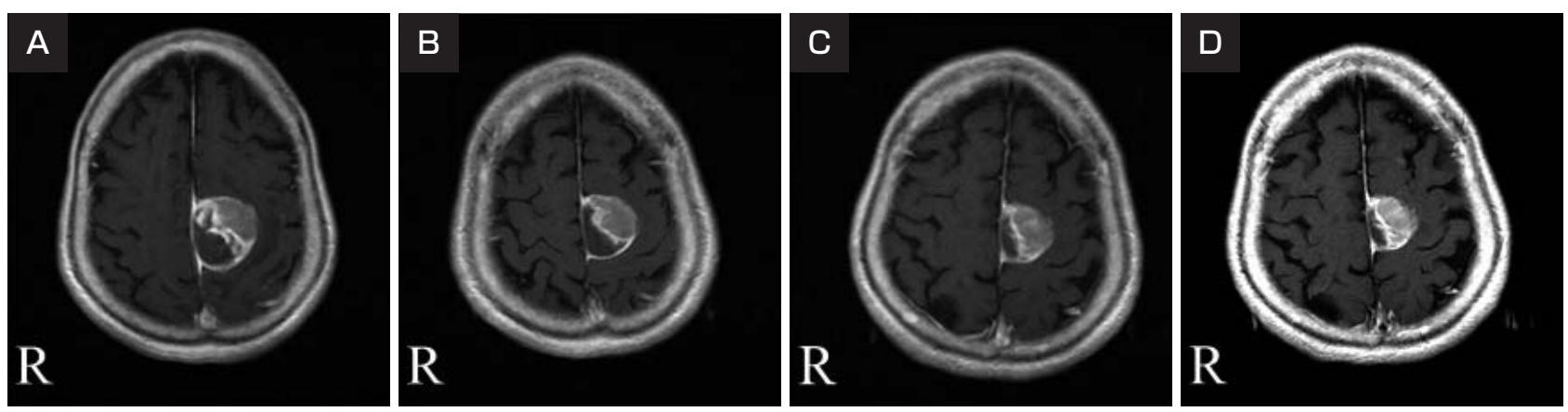

Fig. 4

Axial T1-weighted magnetic resonance images with contrast medium show postoperative tumor shrinkage and improvement of peritumoral edema. Tumor size has not changed in the 2 years since embolization. Images are obtained (A) 6 days, (B) 6 months, (C) 1 year, and (D) 2 years after embolization.

often performed to minimize intraoperative blood loss and facilitate surgical removal ${ }^{9)}$. Several case reports have demonstrated the efficacious treatment of intracranial meningiomas by embolization alone without subsequent surgical removal ${ }^{3-5,14,15,25)}$. Koike et al. and Wakhloo et al. suggested that embolization using polyvinyl alcohol (PVA) was effective for preventing tumor enlargement ${ }^{14,25)}$. Bendszus et al. reported 7 cases of embolization using trisacryl gelatin microspheres that resulted in marked tumor shrinkage over the ensuing follow-up (mean of 20 months) ${ }^{4}$. Kurokawa et al. reported that repeated embolization using absolute ethanol resulted in neurological improvement and tumor shrinkage in the early postoperative period ${ }^{15)}$. Embolization using PVA, gelatin microspheres, or ethanol is relatively uncomplicated because these agents are easy to inject slowly and repeatedly; however, target vessels can recanalize and embolization with small particles carries the risk of intratumoral hemorrhage ${ }^{6,726)}$. Ethanol is superior to other agents used to facilitate vascular penetration because of its low viscosity and high occlusion potential. NBCA is difficult to control during injection because of rapid polymerization, but target vessels rarely recanalize. In this case, we chose ethanol to penetrate to capillary vessels and the strongly emboligenic NBCAlipiodol mixture to avoid recanalization of target vessels and revascularization from the proximal artery. Previous studies of ethanol embolization in other organs have reported rapid necrosis of the target lesion ${ }^{1,13)}$ and brain
MRI revealed tumor shrinkage as early as six days after treatment. This rapid necrotizing effect likely contributed to the improved neurological symptoms of our patient and facilitated timely entry into rehabilitation. Intracranial embolization using ethanol can lead to both systemic and focal complications. Several reports have suggested that the total dose of absolute ethanol should be restricted to less than $1 \mathrm{ml} / \mathrm{kg}$ of body weight to avoid the effects of systemic ethanol toxicity, such as respiratory depression, tachycardia, cardiac arrhythmias, seizures, rhabdomyolysis, and hypoglycemia ${ }^{11,16,23)}$. Previous case studies reported using total volumes of ethanol of 5-55 ml to treat hepatic arteriovenous malformation ${ }^{11,23)}, 8-59 \mathrm{ml}$ to treat pelvic arteriovenous malformation ${ }^{2}, 0.2-0.5 \mathrm{ml} / \mathrm{kg}$ to treat renal cell carcinoma ${ }^{19)}, 20 \mathrm{ml}$ to treat renal angiomyolipoma $^{21)}$ and $3 \mathrm{ml}$ to treat enlarged polycystic kidney ${ }^{20)}$. In the current case, the total volume of ethanol injected was only about $0.6 \mathrm{ml}$, so the chance of toxic side effects was minimal. Venous thrombosis and extravasation are focal complications associated with ethanol injection, but these adverse events can be prevented by placing the microcatheter tip into the feeding artery in the true wedge position and minimizing the volume of ethanol injected. Ethanol was injected only until the tumor contrast agent disappeared. The degree of tumor regression may be hindered by the development of the pial blood supply. Alternatively, if dural blood supply from the middle meningeal artery is dominant, a high tumor regression rate is expected. If tumor size is 
not reduced in the early period after embolization and if the neurological status does not improve, surgical removal should be performed. Reports on intracranial meningioma in the 9th decade of life have found 1-year mortality rates ranging from $9.4 \%$ to $29 \%$, and have suggested that peritumoral edema, tumor location, tumor size, preoperative ASA classification, and preoperative KPS are significant prognostic factors for postoperative morbidity and mortality ${ }^{8,10,18,22)}$. D'Andrea concluded that if the patient's general health is good, open surgery is relatively safe $^{7)}$, but none of these reports examined postoperative cognitive decline. Krupp et al. found a significant negative correlation between patient age and cognitive performance beginning at age 55 years following surgical treatment of supratentorial meningiomas, with particularly serious degradation of concentration and psychomotor abilities ${ }^{16)}$. Therefore, radical surgical treatment should be restricted, especially in cases with low preoperative KPS score or poor general condition, if other options such as endovascular embolization are possible.

\section{Conclusion}

This report describes the case of an 86-year-old woman with intracranial meningioma who was treated solely by endovascular embolization using ethanol and NBCA. Endovascular embolization using liquid may be a better option for symptomatic meningioma patients in poor general condition to avoid postoperative complications such as cognitive impairment.

\section{$<$ Conflict of Interest $>$}

The authors declare no conflicts of interest. This report did not receive any funding from the public or from any commercial agencies.

\section{References}

1) Akasu H, Shimizu K, Kitagawa W, et al: Histological study of papillary thyroid carcinoma treated with percutaneous ethanol injection therapy. Pathol Int 52:406-409, 2002.

2 ) Bae S, Do YS, Shin SW, et al: Ethanol embolotherapy of pelvic arteriovenous malformations: an initial experience. Korean J Radiol 9:148-154, 2008.
3 ) Bendszus M, Klein R, Burger R, et al: Efficacy of trisacryl gelatin microspheres versus polyvinyl alcohol particles in the preoperative embolization of meningiomas. AJNR 21:255-261, 2000.

4) Bendszus M, Martin-Schrader I, Schlake HP, et al: Embolisation of intracranial meningiomas without subsequent surgery. Neuroradiology 45:451-455, 2003.

5 ) Bendszus M, Martin-Schrader I, Warmuth-Metz M, et al: MR imaging-and MR spectroscopy-revealed changes in meningiomas for which embolization was performed without subsequent surgery. AJNR 21:666-669, 2000.

6 ) Bendszus M, Monoranu CM, Schütz A, et al: Neurologic complications after particle embolization of intracranial meningiomas. AJNR 26:1413-1419, 2005.

7 ) Carli DF, Sluzewski M, Beute GN, et al: Complications of particle embolization of meningiomas: frequency, risk factors, and outcome. AJNR 31:152-154, 2010.

8 ) Caroli M, Locatelli M, Prada F, et al: Surgery for intracranial meningiomas in the elderly: a clinicalradiological grading system as a predictor of outcome. $J$ Neurosurg 102:290-294, 2005.

9 ) Chun JY, McDermott MW, Lamborn KR, et al: Delayed surgical resection reduces intraoperative blood loss for embolized meningiomas. Neurosurgery 50:1231-1235, 2002.

10) D’Andrea G, Roperto R, Caroli E, et al: Thirty-seven cases of intracranial meningiomas in the ninth decade of life: our experience and review of the literature. Neurosurgery 56:956-961, 2005.

11) Do YS, Yakes WF, Shin SW, et al: Ethanol embolization of arteriovenous malformations: interim results. Radiology 235:674-682, 2005.

12) Do YS, Park KB, Park HS, et al: Extremity arteriovenous malformations involving the bone: therapeutic outcomes of ethanol embolotherapy. J Vasc Interv Radiol 6:807-816, 2010.

13) Koda M, Okamoto K, Miyoshi Y, et al: Hepatic vascular and bile duct injury after ethanol injection therapy for hepatocellular carcinoma. Gastrointest Radiol 17:167-169, 1992.

14) Koike $T$, Sasaki $O$, Tanaka $R$, et al: Long-term results in a case of meningioma treated by embolization alone--case report. Neurol Med Chir (Tokyo) 30:173-177, 1990.

15) Kurokawa S, Hayashi K, Ogawa S, et al: Embolization therapy without subsequent surgery for falx meningioma; a case report. Rinsho Hoshasen (Japanese) 50:552-557, 2005.

16) Krupp W, Klein C, Koschny R, et al: Assessment of neuropsychological parameters and quality of life to evaluate outcome in patients with surgically treated supratentorial meningiomas. Neurosurgery 64:40-47, 2009.

17) Mason KP, Michna E, Zurakowski D, et al: Serum ethanol levels in children and adults after ethanol embolization or sclerotherapy for vascular anomalies. Radiology 217:127$132,2000$.

18) Mastronardi L, Ferrante L, Qasho R, et al: Intracranial meningiomas in the 9 th decade of life: a retrospective study of 17 surgical cases. Neurosurgery 36:270-274, 1995.

19) Murata S, Tajima H, Onozawa S, et al: Pilot study of transcatheter arterial ethanol embolization under closed 
renal circuit for large renal cell carcinomas. Eur Radiol 18:1464-1472, 2008.

20) Rim H, Jung GS, Jung YS: Transcatheter arterial embolization using ethanol in a dialysis patient for contracting enlarged polycystic kidneys. Korean J Radiol 11:574-578, 2010.

21) Rimon U, Duvdevani M, Garniek A, et al: Ethanol and polyvinyl alcohol mixture for transcatheter embolization of renal angiomyolipoma. AJR 187:762-768, 2006.

22) Sacko O, Sesay M, Roux FE, et al: Intracranial meningioma surgery in the ninth decade of life. Neurosurgery 61:950954, 2007.

23) Senokuchi T, Baba Y, Hayashi S, et al: Embolization of hepatic arteriovenous shunt with absolute ethanol in a patient with hepatocellular carcinoma. Cardiovasc Intervent Radiol 34:154-156, 2010.

24) Unnikrishnan KP, Sinha PK, Sriganesh K, et al: Case report: alterations in bispectral index following absolute alcohol embolization in a patient with intracranial arteriovenous malformation. Can J Anaesth 54:908-911, 2007.

25) Wakhloo AK, Juengling FD, Van Velthoven V, et al: Extended preoperative polyvinyl alcohol microembolization of intracranial meningiomas: assessment of two embolization techniques. AJNR 14:571-582, 1993.

26) Yu SC, Boet R, Wong GK, et al: Postembolization hemorrhage of a large and necrotic meningioma. AJNR 25:506-508, 2004. 